\title{
Spatially Separating Haptic Guidance from Task Dynamics through Wearable Devices
}

\author{
Evan Pezent, Simone Fani, Janelle Clark, Student Members, IEEE, \\ Matteo Bianchi, Member, IEEE, and Marcia K. O'Malley, Senior Member, IEEE,
}

\begin{abstract}
Haptic devices have a high potential for delivering tailored training to novices. These devices can simulate forces associated with real-world tasks, or provide guidance forces that convey task completion and learning strategies. It has been shown, however, that providing both task forces and guidance forces simultaneously through the same haptic interface can lead to novices depending on guidance, being unable to demonstrate skill transfer, or learning the wrong task altogether. This work presents a novel solution whereby task forces are relayed via a kinesthetic haptic interface, while guidance forces are spatially separated through a cutaneous skin stretch modality. We explore different methods of delivering cutaneous based guidance to subjects in a dynamic trajectory following task. We next compare cutaneous guidance to kinesthetic guidance, as is traditional to spatially separated assistance. We further investigate the role of placing cutaneous guidance ipsilateral versus contralateral to the task force device. The efficacies of each guidance condition are compared by examining subject error and movement smoothness. Results show that cutaneous guidance can be as effective as kinesthetic guidance, making it a practical and cost-effective alternative for spatially separated assistance.
\end{abstract}

Index Terms - haptic guidance, training, spatially separated assistance, skin stretch, cutaneous feedback, trajectory following

\section{INTRODUCTION}

$\mathrm{H}$ APTIC training devices have been studied extensively due to the promise of delivering virtual training for real-world tasks which are otherwise too complex, risky, or expensive to train [1], [2], [3]. Haptic, or robot-mediated, training offers a unique set of advantages over traditional human mediated training. First, haptic training devices can record online objective measures of performance in real-time to deliver training protocols tailored to users' skills. Collected data can be used to progressively reduce assistance or increase difficulty, as well as provide realtime performance feedback (e.g. providing movement smoothness information to teleoperators and surgeons [4]). Secondly, haptic devices have the potential to train more users, as a single human expert could conceivably train multiple novices simultaneously, perhaps even remotely. These devices can either be used to create realistic and immersive simulations of training environments, or to deliver forces and cues which convey task completion strategies that accelerate skill acquisition and learning. One area where haptic training devices have flourished is in the medical community [5], particularly in laparoscopic surgery simulation [6] and upperlimb rehabilitation [7]. Haptic training devices can also be found in dentistry [8], manufacturing and assembly [9], and teleoperation [10]. With the advent of commercial virtual and augmented reality systems, it is likely that haptic training will become even more prevalent in the years to come [11].

- E. Pezent, J. Clark, and M. O'Malley are with the Mechatronics and Haptic Interfaces Lab, Dept. of Mechanical Engineering, Rice University, Houston, TX 77005. Email: \{epezent, jc105, omalleym\}@ rice.edu.

- S. Fani is with the Research Center "Enrico Piaggio", and the Dept. of Information Engineering University of Pisa, Largo Lucio Lazzarino 1, 56122 Pisa, Italy, and the Soft Robotics for Human Cooperation and Rehabilitation Research Line, Istituto Italiano di Tecnologia, via Morego, 30, 16163 Genova, Italy.Email: simonefani89@gmail.com.

- M. Bianchi is with the Research Center "Enrico Piaggio", and the Dept. of Information Engineering, University of Pisa, via G. Caruso, 16, 56122 Pisa, Italy.E-mail: matteo.bianchi@centropiaggio.unipi.it.

Manuscript received December 14, 2018; revised Month XX, 2019.
Despite the obvious advantages of haptic training devices, several studies have shown that such training can lead to misinterpretation of feedback [12], a dependence on guidance [13], and an inability to demonstrate skill transfer [14]. The most common reason for this arises from the misconception that simply using physical haptic guidance to assist a novice in completing a task will lead to them being able to complete the task on their own or with increased performance. There is limited evidence for this, and it has been shown that physical guidance may even be detrimental to learning and skill retention [15] since motor learning is mostly thought to be error driven [16]. However, perhaps another reason for the shortcomings of haptic training is in the challenge of reconciling the different types of forces devices should render.

Haptic training forces come in two forms: task forces or guidance forces. Task forces are those which originate from the dynamics of the task or the user's interaction with the environment. In the context of surgical training, for example, a task force might be the collision between the surgical tool and virtual tissue, or the weight of the tool itself. Guidance forces, on the other hand, generally refer to forces which are meant to convey methods of completing the task. Guidance forces may steer a surgeon to avoid certain obstacles or show appropriate interaction forces. Many guidance paradigms have been proposed and compared [14]. Virtual fixtures overlay the task environment with force fields which "push" a novice along a desired trajectory or away from forbidden regions [17]. Contrarily, resistive or error augmenting methods repel users away from a trajectory [18]. Shared control methods allow novices to practice while a virtual "expert" guides them [19]. Regardless of the type of guidance used, one challenge remains: delivering both task forces and guidance forces simultaneously. Specifically, doing so via a single haptic device leads to guidance forces corrupting the novice's perception of task forces, and task forces altering their interpretation of guidance forces. Therefore, it is critical that users correctly discern task and guidance forces for successful training to occur. 


\section{Separating Task and Guidance Forces}

Only a few approaches have been proposed for separating guidance and task forces. Temporally Separated Assistance (TSA) attempts to solve the issue by separating forces on the same haptic channel in time. Trainees experience each force alternately in quick succession with the overall effect being a gradual nudge toward a virtual expert. Endo et al. [20] used this technique to train subjects to grip objects, and Ahn and Hogan [21] showed TSA to encourage different patterns of human gait. Powell and O'Malley implemented TSA for a dynamic target hitting task. In general, they concluded TSA to be sensitive to the timing of guidance force delivery, and confusing and frustrating to users [14].

\subsection{Spatially Separated Assistance}

Another strategy for separating forces is to simply introduce a secondary device or point of contact with the user; one haptic channel is used to deliver task forces, while the other delivers guidance forces. This approach has been categorized as Spatially Separated Assistance (SSA). One of the first examples of SSA was the double-contact paradigm proposed by Gillespie et al. [22]. In their experiment, task forces were delivered through a specialized device on the novice's palm, while guidance forces were delivered on the back of the novice's hand. They hypothesized that this form of guidance would result in greater skill transfer because it allows the novice to discern task and guidance forces while providing as much haptic information as possible. In [23], Wulf et al. demonstrated a form of SSA in a skiing task where guidance was delivered through ski poles, and task forces were delivered through the skis. They showed that this form of practice was superior to practice without guidance. Powell and O'Malley [14] presented a generalized version of SSA where two separate but identical haptic devices were used. Participants controlled a virtual massspring system using a haptic joystick which rendered task forces, while guidance forces were displayed on a secondary joystick on the contralateral hand. They argued that this bimanual approach to training is supported by human motor learning research which suggests partial skill transfer between bimanual and unimanual tasks [24]. Critical for this approach to SSA is that applying forces to one arm will not impede learning of force fields by another arm, as shown by Techean et al. [25].

In each of these examples of SSA, complex and potentially expensive kinesthetic type haptic hardware was developed specifically for the task (other examples include rowing [26], [27] and tennis training [28] hardware). Thus, this form of force separation is both impractical and difficult to generalize. Furthermore, while some types of kinesthetic haptic devices have been shown to enhance performance, many have been ineffective when it comes to demonstrating skill retention and transfer [14], [29], [30].

\subsection{Cutaneous and Skin-Stretch Haptic Devices}

The past decade has seen paradigm shift in the haptics community from expensive, grounded kinesthetic type devices to low-cost, wearable cutaneous type devices [31]. These devices target skin sensory channels, namely those which detect pressure, stretch, and vibration. In contrast to specialized kinesthetic devices, cutaneous devices are widely applicable to training complex movements, can be generalized to different tasks, are inherently safer, and harmoniously integrate with the human body [32]. Tactile cueing systems have been extensively studied to determine appropriate methods for guiding wrist rotation movements [33], and motion guidance has been effectively conveyed through both vibrational [34] and skin stretch [35] tactile cues. Ruffaldi [36] showed that learning an abstract trajectory on a rowing simulator was slightly more enhanced by visuo-vibrotactile feedback than by visual or vibrotactile feedback alone. Researchers have also effectively combined cutaneous devices with kinesthetic devices in the fields of teloperation and robotic surgery [37], [38]. As a form of SSA, Peon et al. [39] utilized kinesthetic feedback for tissue rendering, and vibrational feedback as a virtual fixture type corrective system.

Skin stretch (or shear) is particularly interesting in the context of training, as Kim and Colgate [40] have hypothesized that skin stretch delivers directional information through a modality matching approach similar to how a human trainer might guide a trainee's movement. Skin stretch devices may utilize bands or rockers to deliver stretch longitudinally, latitudinally, rotationally, or circumferentially on various parts of the body.

This paper presents a novel form of SSA whereby an existing kinesthetic training platform is supplemented with a wearable cutaneous device. Task forces are relayed via the kinesthetic haptic interface at the hand, while guidance forces are conveyed through skin stretch on the arm (Fig. 1). This modular approach to SSA provides several advantages over the traditional method of introducing a second kinesthetic device for guidance, particularly in terms of being less expensive, more practical, and more easily generalized to already existing training devices and tasks.

We investigate this approach to SSA using a simple dynamic trajectory following task, described in Section 3, which was administered to subjects across five guidance conditions. In Section 4, we explore two methods of providing guidance with the proposed device. Specifically, we compare performance dependent, or feedback based, guidance with performance independent, or feedforward based, guidance. Next, in Section 5 . we extend our work from [41] where we directly compare the effectiveness of SSA achieved via our cutaneous approach and traditional kinesthetic guidance, and further explore the role of placing cutaneous guidance ipsilateral versus contralateral, as is necessary when using a kinematically similar kinesthetic device. In addition to expounding upon our previous analysis, we provide new analysis of subject performance which examines movement smoothness and trajectory characteristics in the frequency domain. A discussion of all experiments can be found in Section 6 with conclusions and future work following in Section 7 .

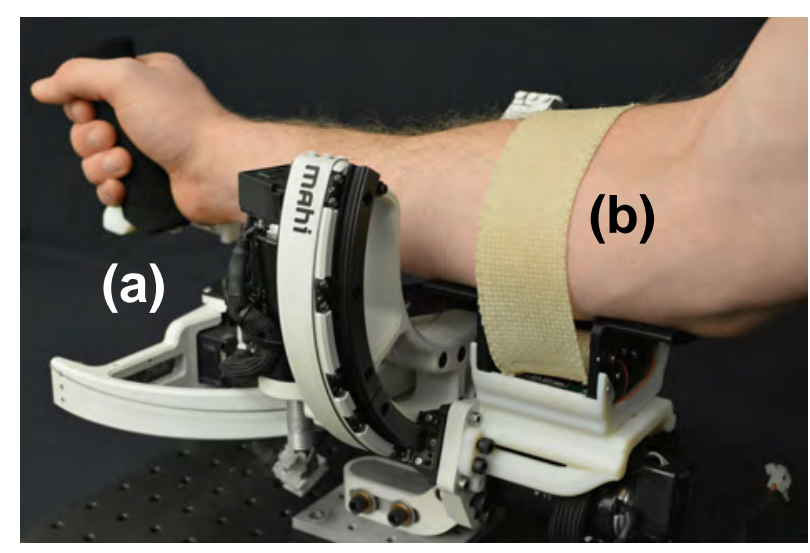

Figure 1. Separating task and guidance forces during haptic training remains a fundamental issue in the field. This paper presents a novel approach in which (a) an existing kinesthetic device displaying task forces at the hand has been outfitted with (b) a new cutaneous skin stretch device to provide spatially separated guidance forces on the arm. 


\section{Materials and Methods}

We designed a trajectory following task, displaying pertinent and non-negligible dynamic forces. Subjects used their right arm to complete the task through positional input of a wrist-forearm exoskeletal device (the Task Device), which also rendered task forces. In our first experiment (Section 4), a skin-stretch device (the Cutaneous Guidance Device) was used to provide guidance on the arm ipsilateral to the Task Device. In the second experiment, (Section 5, a kinematically similar exoskeleton (the Kinesthetic Guidance Device) provided proprioceptive guidance on the contralateral arm and was compared with the Cutaneous Guidance Device in both ipsilateral and contralateral configurations. Here, we describe the hardware and task common to both experiments.

\subsection{Task Device}

The OpenWrist [42] was chosen as the task completion device (Fig. 2p. This choice should be considered arbitrary, as other kinesthetic haptic interfaces could have served this role. The OpenWrist is a 3 degree-of-freedom (DOF) wrist exoskeleton designed for wrist rehabilitation, capable of rendering haptic environments through the application of DC motors and capstancable transmissions. The first joint of the exoskeleton, forearm pronation/supination (PS), was used as the driving input of the task, while the two distal joints, wrist flexion/extension (FE) and radial/ulnar deviation (RU), were locked in their neutral positions through control. Gravity and Coulomb friction compensation torques were added to the task torques so that the dominant torques felt by the user would arise from the dynamics of the virtual task.

\subsection{Kinesthetic Guidance Device}

The MAHI Exo-II (ME-II) [44] upper extremity exoskeleton was used for the kinesthetic guidance condition (Fig. 4). Like the OpenWrist, the ME-II employs a DC motor and capstan-cable transmission to achieve forearm rotation. Proprioceptive guidance was provided through the rotation of the forearm PS joint. It is important to note that the ME-II differs from the OpenWrist in that it uses a parallel prismatic mechanism for its FE/RU wrist joints, and provides an additional joint for the elbow. Because these joints were locked either mechanically or through control for the duration of the experiment, the kinematic differences between the OpenWrist and ME-II were considered negligible.

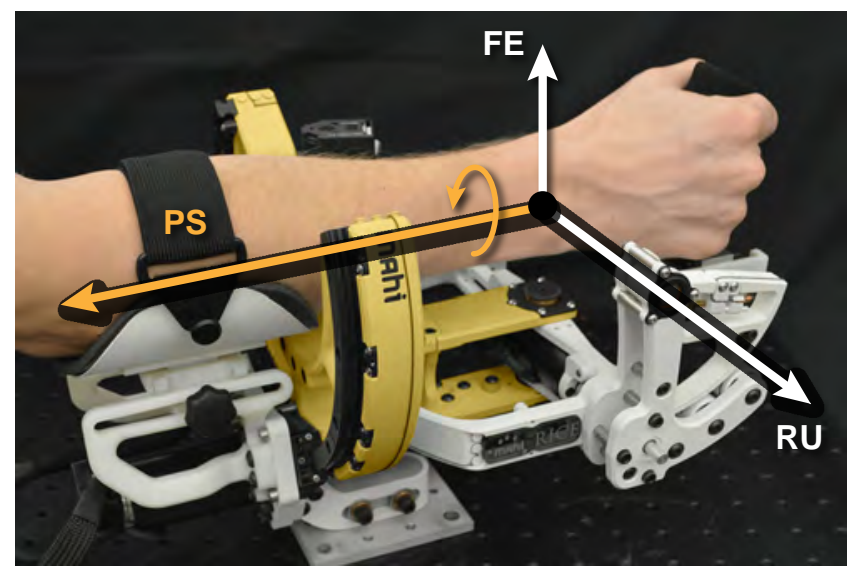

Figure 2. The OpenWrist [42 served as the Task Device. The forearm pronation/supination (PS) DOF (yellow) was used for task input and rendering task forces, while the wrist flexion/extension (FE) and radial/ulnar (RU) deviation DOFs were locked in their neutral positions.

\subsection{Cutaneous Guidance Device}

For cutaneous guidance, we used the Clenching Upper-limb Force Feedback device (CUFF), a wearable haptic device $(123.5 \times 76 \times 80 \mathrm{~mm}, 226 \mathrm{~g})$ that distributes mechano-tactile forces on the user's skin [43] and has been successfully employed in prosthetic and augmented human-robot applications [45], [46] (Fig. 3). The device consists of a composite silicone-fabric band which is wrapped around the user's limb. Two DC motors, attached to opposite ends of the band, are independently driven in either opposite or same directions to display normal or tangential cutaneous force, respectively. For consistency, in this application the band is pretensioned to constantly exert a normal force of $3 \mathrm{~N}$ against the arm, and only the tangential cutaneous force is used.

While the CUFF is typically worn as an ungrounded device, it was fixed to the work surface in both experiments. In the ipsilateral configuration, it was directly integrated with the OpenWrist Task Device, replacing the default forearm rest (Fig. 11, and in the contralateral configuration, it was mounted to a frame of the same height (see Fig. 11). A pillow hand rest was provided to prevent fatigue in the contralateral configuration.

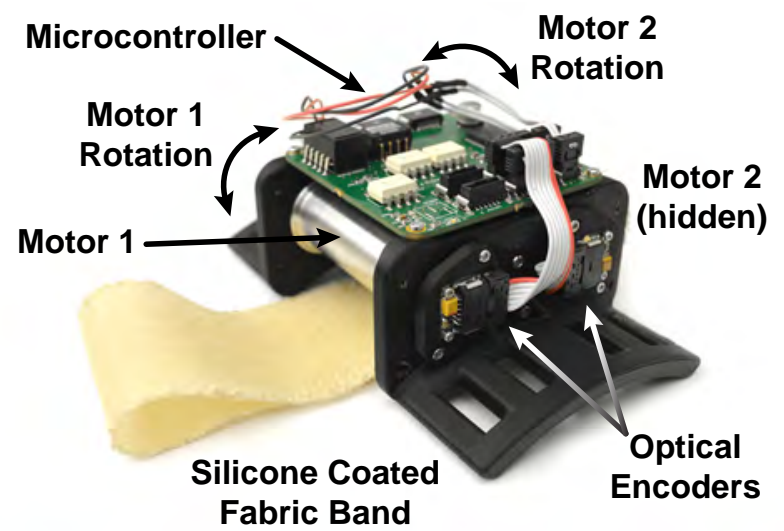

Figure 3. The Clenching Upper-limb Force Feedback device (CUFF) [43 served as the Cutaneous Guidance Device. Two DC motors attached to rollers tension a silicone coated fabric band to create skin stretch sensations on the user's arm. Although typically worn as an upper arm band, the CUFF was fixed during our experiments.

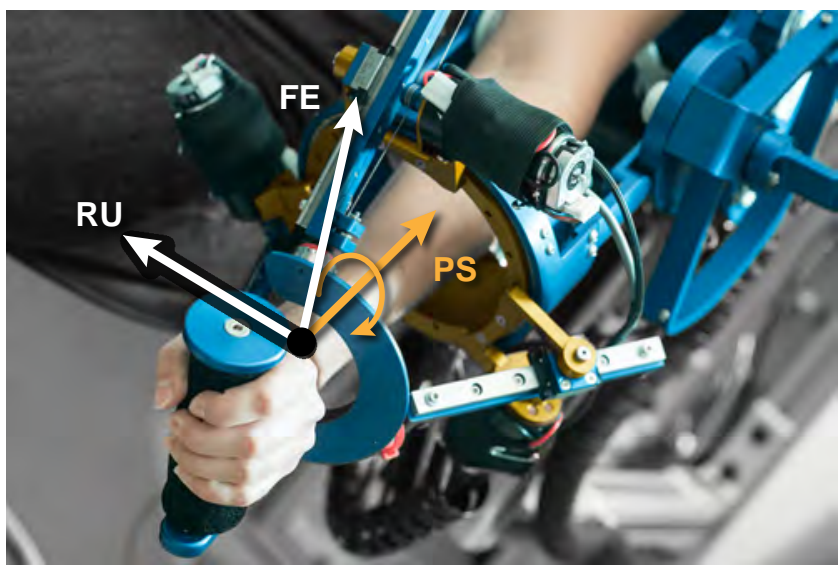

Figure 4. The MAHI Exo-II [44] was the Kinesthetic Guidance Device. The elbow DOF and parallel mechanism providing flexion/extension (FE) and radial/ulnar deviation (RU) were locked in a neutral position, while the forearm pronation/supination (PS) DOF (yellow) provided guidance. 


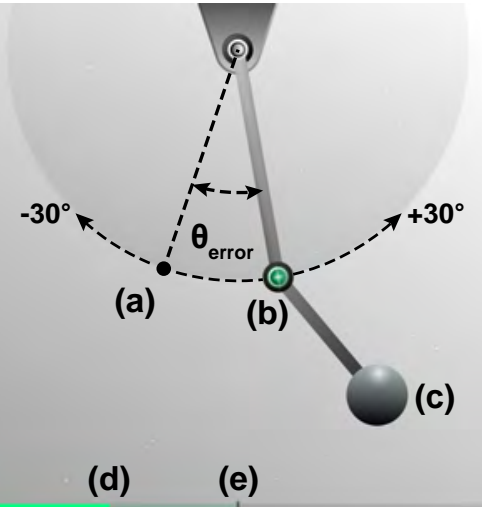

Figure 5. Task Visualization - The angular reference trajectory, represented by the black expert dot (a) moves along the dashed arc. Note that the expert dot is shown to the user during a familiarization period, but hidden from view in all subsequent trials. The subject rotates the first pendulum link, moving the location of the indicator light (b). The indicator light intensity increases as $\theta_{\text {error }}$ shrinks. Thus, the objective was to keep the light shining as brightly as possible at all times. The second, freely rotating pendulum link and attached mass, (c) contribute to dynamic task forces. The score bar (d) fills as the user maintains close proximity to trajectory reference angle, and a tic mark (e) shows the user's previous high score for the current trajectory.

\subsection{Description of Task}

Consideration should be taken when designing a controlled task that combines haptic guidance and task dynamics with visual information. Forces should be physically relevant to the displayed visuals, and the task should necessitate haptic guidance, proving difficult or impossible without it. Furthermore, the task must be intuitive for first-time users, yet provide enough challenge so that learning occurs.

In this experiment, subjects were tasked with controlling the position of a virtual double pendulum (Fig. 5) displayed on a computer screen. The pendulum, with its first joint coupled to the OpenWrist forearm PS joint via a stiff virtual spring-damper, was simulated by numerically integrating

$$
\begin{gathered}
\tau_{1}=K\left(\theta_{\text {ow }}-\theta\right)+B\left(\dot{\theta}_{\text {ow }}-\dot{\theta}\right)= \\
{\left[l_{1}^{2} m_{1}+\left(l_{1}^{2}+l_{2}^{2}+2 l_{1} l_{2} \mathrm{c}_{2}\right) m_{2}\right] \ddot{\theta}_{1}+b_{1} \dot{\theta}_{1}} \\
+\left(l_{2}^{2}+l_{1} l_{2} \mathrm{c}_{2}\right) m_{2} \ddot{\theta}_{2}-l_{1} l_{2} \mathrm{~s}_{2} m_{2} \dot{\theta}_{2}^{2}-2 l_{1} l_{2} \mathrm{~s}_{2} m_{2} \dot{\theta}_{1} \dot{\theta}_{2} \\
+\left[l_{1} \mathrm{c}_{1} m_{1}+\left(l_{1} \mathrm{c}_{1}+l_{2} \mathrm{c}_{1+2}\right) m_{2}\right] g \\
\tau_{2}=\left(l_{1} l_{2} \mathrm{c}_{2}+l_{2}^{2}\right) m_{2} \ddot{\theta}_{1}+l_{1} l_{2} \mathrm{~s}_{2} m_{2} \dot{\theta}_{1}^{2} \\
+l_{2}^{2} m_{2} \ddot{\theta}_{2}+b_{2} \dot{\theta}_{2}+l_{2} \mathrm{c}_{1+2} m_{2} g=0
\end{gathered}
$$

where $\theta_{1}, \theta_{2}$, and their derivatives are the pendulum state, $s_{i}$ is $\sin \theta_{i}, \mathrm{c}_{i}$ is $\cos \theta_{i}, \tau_{1}$ and $\tau_{2}$ are the pendulum joint torques, and $\theta_{o w}$ and $\dot{\theta}_{o w}$ are the position and velocity of the OpenWrist forearm PS joint. The coupling stiffness $K$ and damping $B$ were $15 \mathrm{Nm} / \mathrm{rad}$ and $1 \mathrm{Nm}$-s/rad, respectively. While maintaining control of the first link, subjects could feel the pendulum reaction torque $\tau_{1}$, which was rendered with the OpenWrist. The pendulum parameters $\left(m_{1}=10 \mathrm{~g}, m_{2}=150 \mathrm{~g}, l_{1}=45 \mathrm{~cm}, l_{2}=30 \mathrm{~cm}\right.$, and $b_{1}=b_{2}=1 \mathrm{mN} \mathrm{ms} \mathrm{rad}^{-1}$ ) were chosen during pilot trials so that the pendulum was relatively dynamic, but not fatiguing for subjects to handle. In this way, our task design provided meaningful forces that were easily understood by the user, while remaining non-negligible.
While in control of the double pendulum, users were asked to follow a continuous angular trajectory $\theta_{\text {ref }}$ with the first link of the pendulum. The equation for the reference trajectory was computed through the summation of sine waves (Eqn. 3) and further normalized (Eqn. 4) to an amplitude of \pm 30 degrees, the largest forearm rotation that would not result in subject fatigue. Three unique sets of equation parameters (Table 1) were chosen to represent Easy, Medium, and Hard trajectories, shown in Fig. 6 Parameters were selected and tested during pilot trials under the intuition that increasing the frequency and number of frequency modes would elevate difficulty. Thus Easy has one mode, Medium has two modes, and Hard has three modes.

$$
\begin{gathered}
\theta_{r e f}^{\prime}(t)=A \sin (2 \pi a t)+B \sin (2 \pi b t)+C \sin (2 \pi c t) \\
\theta_{\text {ref }}(t)=30^{\circ} \frac{\theta_{r e f}^{\prime}(t)}{\max \theta_{r e f}^{\prime}}
\end{gathered}
$$

The trajectory itself was not directly displayed to the subject, since this would have trivialized the task. Instead, a visual indicator in the form of a light on the second pendulum joint indicated proximity to the trajectory reference angle. The brightness of the light increased as the subject approached the reference angle, or decreased their angular error. Thus, in the visual absence of the reference trajectory, the subject's objective was to keep the light shining as brightly as possible throughout each trial.

The visual feedback from the pendulum indicator light alone did not facilitate successful completion of the task. While it provided proximal feedback, it did not convey which direction to move, thus necessitating haptic guidance.

To keep subjects engaged, a score bar was shown at the bottom of the screen. The scoring formula, given by

$$
\text { score }=\sum_{t=0}^{T} \max \left(0, \min \left(10,10-\left|\theta_{\text {error }}(t)\right|\right)\right)
$$

where $\theta_{\text {error }}(t)=\theta_{\text {ow }}(t)-\theta_{\text {ref }}(t)$ is calculated in degrees, rewarded players for remaining below $10^{\circ}$ of error, a challenging but feasible feat. The score bar was reset with each new trial, and the subject's high score for the current trajectory was shown to encourage continuous improvement.

Table 1 Trajectory Parameters

\begin{tabular}{lcccccc} 
Difficulty & $\mathbf{A}$ & $\mathbf{B}$ & $\mathbf{C}$ & $\mathbf{a}[\mathbf{H z}]$ & $\mathbf{b}[\mathbf{H z}]$ & $\mathbf{c}[\mathbf{H z}]$ \\
\hline Easy & 1.0 & 0.0 & 0.0 & 0.20 & 0.0 & 0.0 \\
Medium & -1.0 & -0.5 & 0.0 & 0.25 & 0.1 & 0.0 \\
Hard & 0.5 & -0.5 & -1.0 & 0.20 & 0.1 & 0.4
\end{tabular}

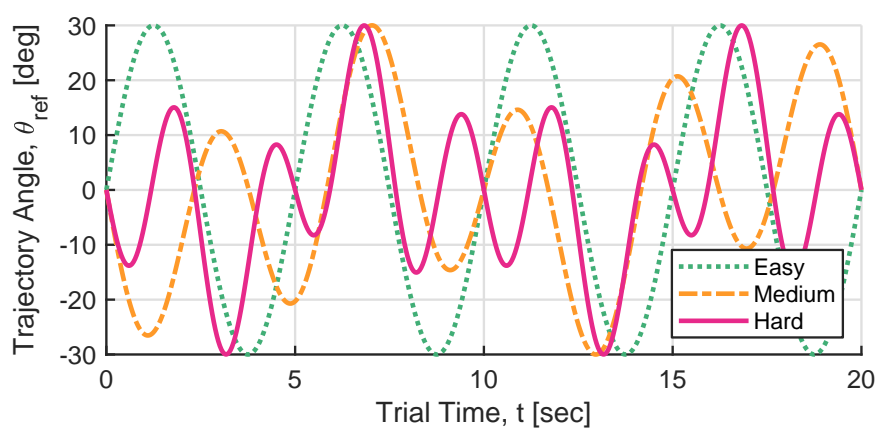

Figure 6. The angular trajectories subjects were required to follow. Harder trajectories are characterized by additional frequency modes. 


\section{Cutaneous Guidance Paradigms}

In this section we discuss our initial investigations into which paradigms of cutaneous guidance would be most effective in a trajectory following context. Specifically, we wanted to answer whether feedforward guidance or feedback guidance would help users follow the trajectory more precisely.

In the feedforward scheme, the magnitude of guidance cues was proportional to the trajectory angle. Specifically, both motors rotated in the same direction to generate tangential forces on the subject's forearm. In essence, the trajectory is rendered directly on the user's skin. Note that the CUFF forces were not sufficient to cause rotation of the forearm or Task Device, nor did voluntary forearm rotation significantly affect the consistency of CUFF forearm skin stretch. The motor position gain (250 ticks/deg) was selected so that the band rotation would approximately match the angular trajectory, as we felt that this would alleviate discrepancies between the task and guidance coordinates.

In the feedback scheme, the magnitude of guidance cues was proportional to the error between the subject's position and the trajectory. This scheme was implemented such that if the subject should rotate their arm counterclockwise to correct their error, the band would also stretch forearm skin counterclockwise, and vice versa. Thus, if a subject were to follow the trajectory precisely, they would experience minimal to no skin stretch.

\subsection{Experimental Design}

The study was performed during one-hour sessions which consisted of a two minute familiarization period and 72 experimental trials, each lasting twenty seconds. Each subject received only one of the three guidance conditions:

FF Feedforward CUFF guidance on the ipsilateral arm

FB Feedback CUFF guidance on the ipsilateral arm

V No haptic guidance; visuals only

In conditions FF and FB, the CUFF was integrated directly into the Task Device (Fig. 11). A curtain occluded the subject's view of the CUFF to prevent unintended use of visual information.

As in [14], subjects were given a brief familiarization phase. They were shown the reference trajectory as an on-screen expert dot (Fig. 5-a), and were instructed to internalize the relationships between the dot location and the guidance sensation, indicator light, and score bar. The trajectory used in this phase was different from those given in Table 1 Participants were told that in future trials, the expert dot would be hidden, and they would be required to find the path based solely on the haptic guidance and the indicator light. After familiarization, subjects completed six blocks of trials. Each block consisted of four Easy, four Medium, and four Hard trials shuffled randomly. Depending on which trajectory was presented, the pendulum indicator light and score bar would change color to green (Easy), yellow (Medium), or red (Hard) so that subjects could learn the different trajectories as separate entities. After 36 trials, subjects were allowed to take a five minute break and then returned for another three blocks of training.

\subsection{Subjects}

A total of 30 subjects ( 6 female, ages mean \pm SD: $22.6 \pm 4.2$ ) were enrolled in the experiment and were divided evenly among the three experimental conditions. All subjects were right-handed with no significant visual or motor impairments, and had limited or no experience with skin-stretch haptic devices. Each subject provided informed consent according to the policies of the the Rice University Institutional Review Board (IRB-FY-2018-29).

\subsection{Data Analysis}

Participant performance was quantified with two variables to assess how well they completed the task and the smoothness of their control. The first was the mean absolute error between the subject's trajectory and the expert trajectory, which served to quantify their similarity. Dynamic Time Warping was also considered for comparing similarity, however this method tends to remove temporal information and would hide discrepancies in phase lag between the conditions. The second measure was Spectral Arc Length (SPARC) [47], which has been used within the rehabilitation robotics community. SPARC is the length of the Fourier magnitude spectrum of a movement's velocity profile. It takes on dimensionless negative values, with less negative values indicating smoother movements. We used the MATLAB function provided by SPARC's authors with the default settings.

Both metrics were evaluated to compare the effectiveness of guidance conditions in improving participant performance during the task. Three factors were included in the analysis. Block refers to the error and smoothness for each of the six training blocks within the experiment. Difficulty pertains to the three difficulty levels within the experiment, Easy, Medium, and Hard. The third factor, Condition, compares visual-only, feedforward or feedback conditions. A $3 \times 6 \times 3$ [Condition $(\mathrm{V}, \mathrm{FB}, \mathrm{FF}) \times$ Block $(\mathrm{B} 1, \mathrm{~B} 2$, B3, B4, B5, B6) $\times$ Difficulty (Easy, Medium, Hard)] ANOVA, with repeated-measures on the last two factors, was conducted. Using a three inter-quartile range (IQR) criteria, data was checked for outliers: first on each within-subject data condition separately, and next on the between subject factor of Condition. No outliers were found. Sphericity violations were treated with a HuynhFeldt adjustment as needed. Seven additional comparisons were analyzed using a sequential Bonferroni adjustment. A t-test was used to compare B1 and B6 to assess net improvement after completing the experiment. A one-way ANOVA was used for B1 and B6 individually, with additional contrasts to compare $\mathrm{V}$ and $\mathrm{FB}$, as well as comparing FF against both FB and V.

\subsection{Results}

Fig. 7 gives a high level overview of how subjects' performance progressed throughout the experiment. Here, all subjects' Easy trials are averaged with condition and block. For reference, the actual trajectory is shown as a dotted line. In Block 1, we see minimal difference between conditions $\mathrm{V}$ and FB, and both show little resemblance to the actual trajectory and also exhibit a high degree of jerkiness. Contrarily, the FF condition has already begun to take the shape of the actual trajectory and is considerably smoother than V and FB. From Blocks 2 to $6, \mathrm{~V}$ and FB slightly conform to the trajectory, but continue to show jerkiness and large standard deviation. FF, however, shows drastic improvements with the average trajectory beginning to overlap the actual trajectory in Block 3, and standard deviation reaching its lowest point in Block 6. The other difficulties generally show the same phenomenon, with conditions being somewhat less distinguishable for Hard.

To further investigate differences in subjects' performance, consider the single sided amplitude spectrum of selected subject trajectories from the FF group in Block 6 (Fig. 8). Here, the solid black line represents the actual trajectory, where peaks correspond to the frequencies and amplitudes of the underlying sinusoidal components given in Table 1 Generally, subjects who performed well could identify each frequency and amplitude, even on the Hard difficulty. Average subjects could identify the high amplitude 


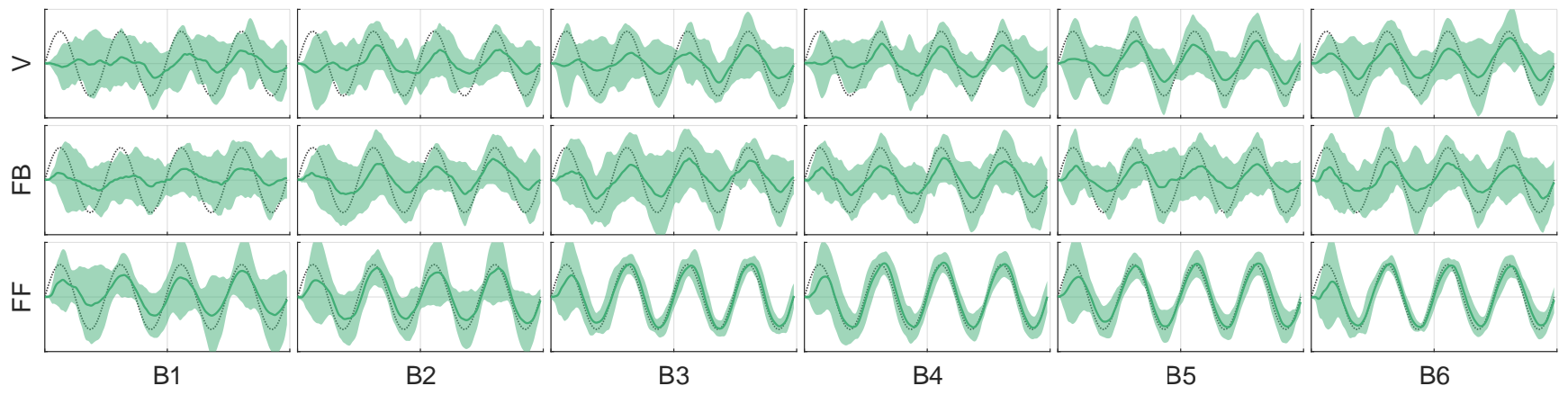

Figure 7. Average of Easy trajectories across all subjects grouped by block and condition. The shaded regions shows the standard deviation of the mean. The dotted line represents the true trajectory subjects should follow. Trajectories from subjects in the visuals only (V) and feedback conditions (FB) exhibit similar characteristics, namely large standard deviation, jerkiness, and little improvement from B1 to B6. Feedforward guidance (FF) however begins lower starting error and shows continuous improvement until the point of closely overlapping the true trajectory in B6.
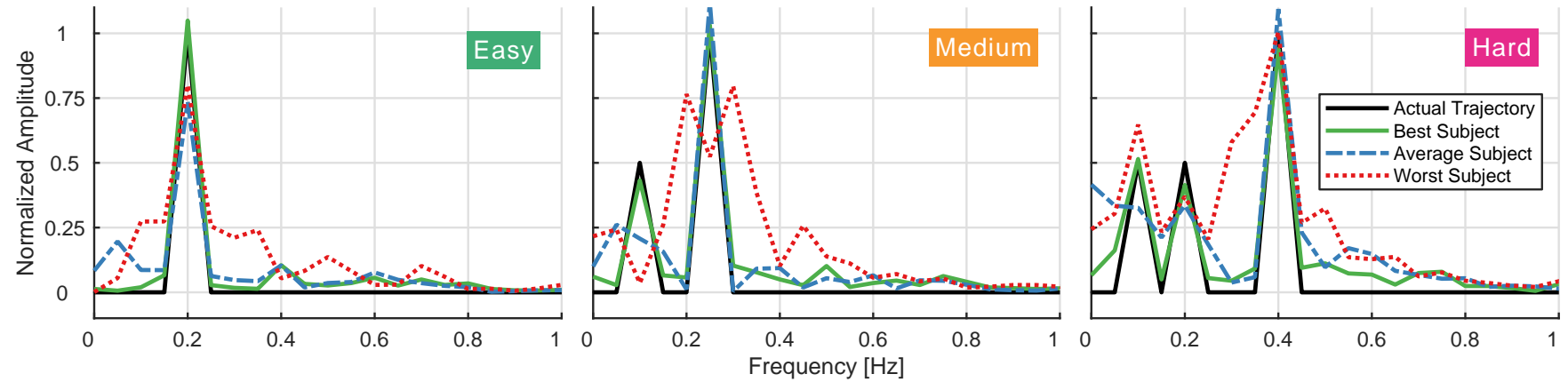

Figure 8. Single sided amplitude spectrum of selected subject trajectories from the feedforward condition for all difficulties. The peaks in the Actual Trajectory (solid black) correspond with the values in Table 1 Even on the hardest difficulty, skilled subjects can identify each frequency and amplitude. Average subjects have difficulty identifying low amplitude frequencies, and the poorest performing subjects exhibit off-frequency content.

frequency, but not always the low amplitude frequency. Poor performing subjects might not identify any frequencies, and almost always displayed significant off-frequency content.

The variations in participant performance are best visualized using the data for the Easy task, as shown in Fig. 7 though there was not a significant difference in Difficulty for each of the performance metrics. There was an effect across Block $(F(3.95,106.5)=$ $19.81, \mathrm{p}<.001)$ and Condition $(F(2,27)=7.06, \mathrm{p}=.003)$, more clearly shown in Fig. 9. To capture learning, the initial and final blocks were compared, showing a significant difference in error between B6 and B1 $(t(29)=8.07, \mathrm{p}<.001)$. The differences in Condition, both at the beginning and end of the experiment, were significant, for B6 $(F(2,27)=9.29, \mathrm{p}=.001)$ and $\mathrm{B} 1(F(2,27)=$ $5.44, \mathrm{p}=.01)$. Breaking this down further, more error was incurred in conditions $\mathrm{V}$ and $\mathrm{FB}$ compared to $\mathrm{FF}$ in $\mathrm{B} 6(F(1,27)=18.33$, $\mathrm{p}<.001)$ as well as B1 $(F(1,27)=10.68, \mathrm{p}=.003)$. However, no significant difference between $\mathrm{V}$ and $\mathrm{FB}$ was present.

The results for smoothness are similar to those for mean absolute error. The main effects show that Block does have a significant effect $(F(2.19,59.2)=10.42, \mathrm{p}<.001)$, as well as the Condition $(F(2,27)=7.69, \mathrm{p}=.002)$, though no effect of Difficulty. In Fig 10, we can again see improvement in SPARC between the first and last Block $(t(29)=3.81, \mathrm{p}=.001)$. Within each of those blocks, there are significant differences in the Condition for B6 $(F(2,27)$ $=6.57, \mathrm{p}=.005)$ and also B1 $(F(2,27)=6.70, \mathrm{p}=.004)$. Subjects who received the feedforward guidance displayed significantly smoother trajectories than those who received feedback or no guidance at all for B6 $(F(2,27)=12.59, \mathrm{p}=.001)$ and $\mathrm{B} 1(F(2,27)$ $=10.95, \mathrm{p}=.003)$. Again, there is little to distinguish $\mathrm{V}$ from $\mathrm{FB}$, with there being no significant difference in either B6 or B1.

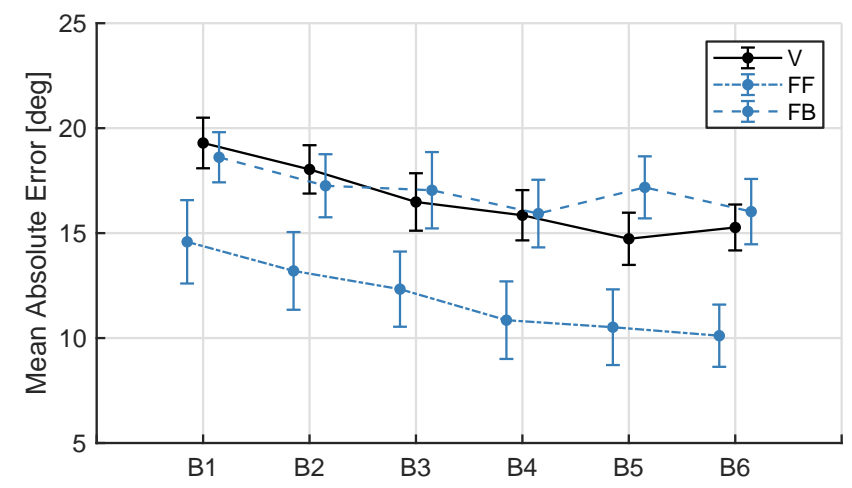

Figure 9. Mean Absolute Error averaged across all difficulties, grouped by block and condition. Subjects with feedforward guidance condition (FF) performed significantly better than those in the feedback guidance FB and visual only (V) conditions.

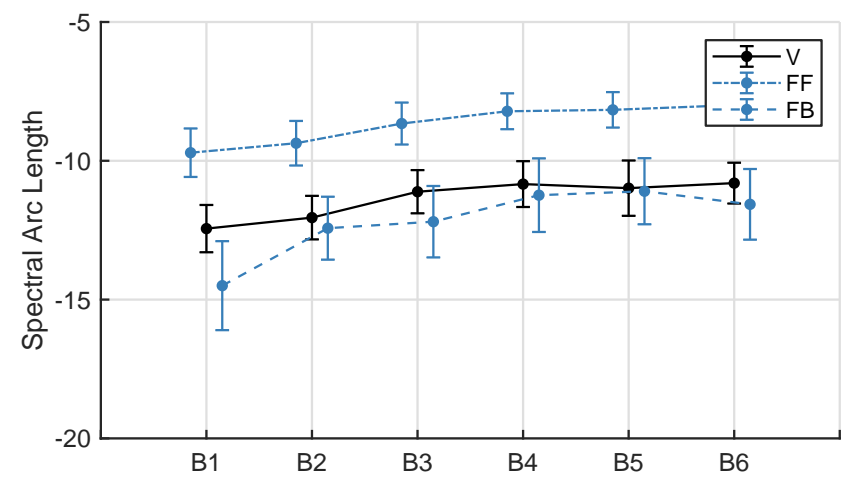

Figure 10. Spectral Arc Length averaged across all difficulties, grouped by block and condition. Less negative values indicate smoother movements. Feedforward guidance (FF) produced significantly smoother movements than feedback guidance (FB) and no guidance (V). 

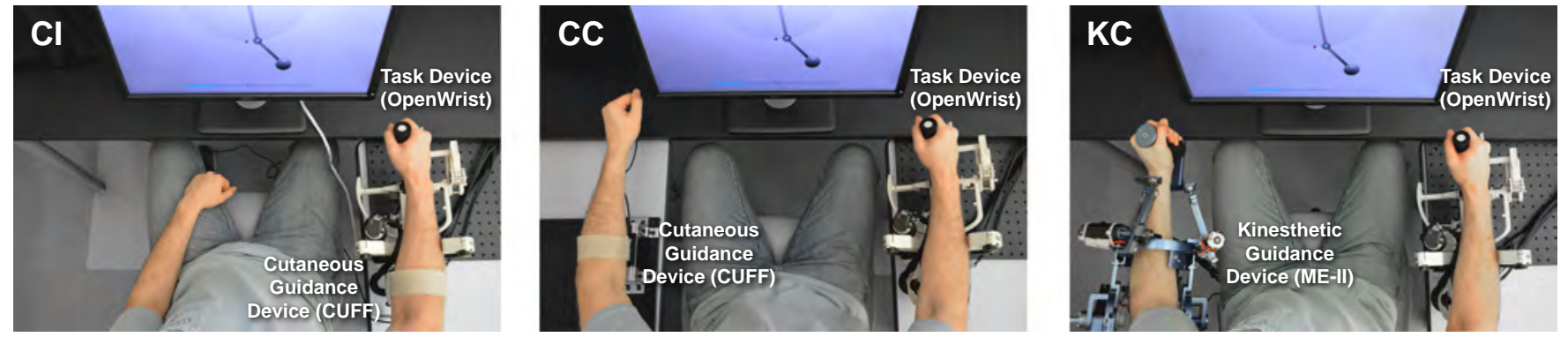

Figure 11. Guidance Conditions - (CI) cutaneous guidance device (CUFF) on the ipsilateral arm, mounted to the OpenWrist, (CC) cutaneous guidance device (CUFF) on the contralateral arm, mounted to an 80-20 frame. (KC) kinesthetic guidance device (MAHI Exo-II) on the contralateral arm, In each condition, the OpenWrist Task Device is used with the right arm, and the subject's view of the guidance device is obstructed with a curtain (not shown).

\section{Cutaneous vs. Kinesthetic Guidance}

Based on the results presented in Section 4 , we determined the feedforward paradigm as the best candidate for delivering cutaneous guidance. We next sought to compare cutaneous guidance with kinesthetic guidance for SSA. We therefore introduced the ME-II to deliver feedforward kinesthetic guidance on the contralateral arm (shown in right image of Fig. 111) in a new experimental condition.

We also explored the differences between placing cutaneous guidance ipsilateral versus contralateral, as is necessary when using the kinematically similar kinesthetic guidance device. Thus we introduced a second new condition where the CUFF was removed from the OpenWrist and mounted to an elevated frame for the contralateral arm as shown in the center of Fig. 11 .

Similar to the CUFF in feedforward mode, the forearm DOF of the ME-II followed the trajectory reference angle through position control. It is important to discuss the choice of position-controlled guidance. With the CUFF, motor positions are commanded to move the belt which, due to contact friction and skin elasticity, imparts a shear force. Consequently, we decided that the ME-II should also employ position control, rather than torque control, in order to maintain consistency across guidance conditions. Furthermore, torque guidance would be counterintuitive since the ME-II's forearm joint angle would inadvertently drift over time, causing confusion for any subjects who might be relying on proprioceptive information. To mimic the elastic nature of the CUFF-skin interaction, the PD position controller on the ME-II was tuned to display a low impedance $\left(K_{P}=4 \mathrm{Nm} / \mathrm{rad}, K_{D}=\right.$ $0.05 \mathrm{Nm}-\mathrm{s} / \mathrm{rad}$ ), which also minimized subject discomfort.

\subsection{Experimental Design}

A total of three separate haptic guidance conditions were considered for this part of experiment:

CI Cutaneous ipsilateral guidance from the CUFF

CC Cutaneous contralateral guidance from the CUFF

KC Kinesthetic contralateral guidance from the ME-II

Conditions $\mathbf{K C}$ and $\mathbf{C C}$ are the newly introduced conditions, and condition $\mathbf{C I}$ is reused from condition $\mathbf{F F}$ in the initial part of our experiment. Again, a curtain was used to occlude the subjects' view of the guidance devices. All other aspects of the experiment remained the same as described in Section 4.1

\subsection{Subjects}

An additional 20 subjects were recruited to fill the $\mathbf{K C}$ and $\mathbf{C C}$ conditions. Combined with subjects from the CI condition, the demographic distribution was 22 male, 8 female, ages $20.2 \pm 0.6$ (mean $\pm \mathrm{SD}$ ), all right handed, and no visual deficiencies.

\subsection{Data Analysis}

The absolute mean error and SPARC were again used to assess participant performance in the trajectory-following task. The same six Block and three Difficulty structures was present, though the Condition compares contralateral, ipsilateral, and kinesthetic feedback. A $3 \times 6 \times 3$ [Condition (CC, CI, KC) $\times$ Block (B1, B2, B3, B4, B5, B6) $\times$ Difficulty (Easy, Medium, Hard)] ANOVA, with repeated-measures on the last two factors, was conducted. Sphericity violations were treated with a Huynh-Feldt adjustment as needed. Seven additional comparisons were analyzed using a sequential Bonferroni adjustment. A t-test was used to compare B1

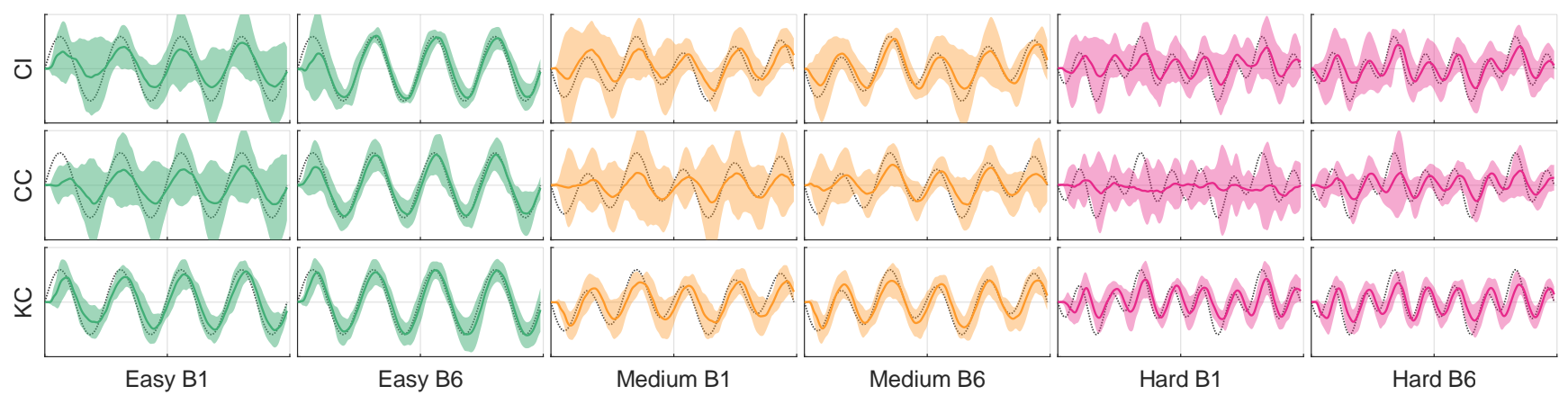

Figure 12. Average trajectories across all subjects near the beginning (B1) and end (B6) of the experiment for conditions $\mathrm{Cl}$, CC, and $\mathrm{KC}$. In all difficulties, subjects in the cutaneous conditions $\mathrm{Cl}$ and $\mathrm{CC}$ begin with lower accuracy and higher standard deviation when compared with the kinesthetic condition KC. However, by B6 their error and standard deviation decrease to levels closer to the KC condition, especially for the Easy and Medium difficulties. 


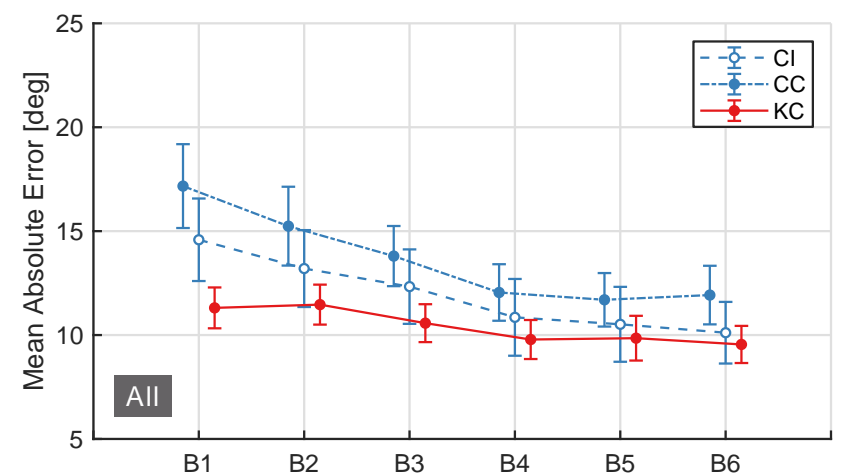

(a)

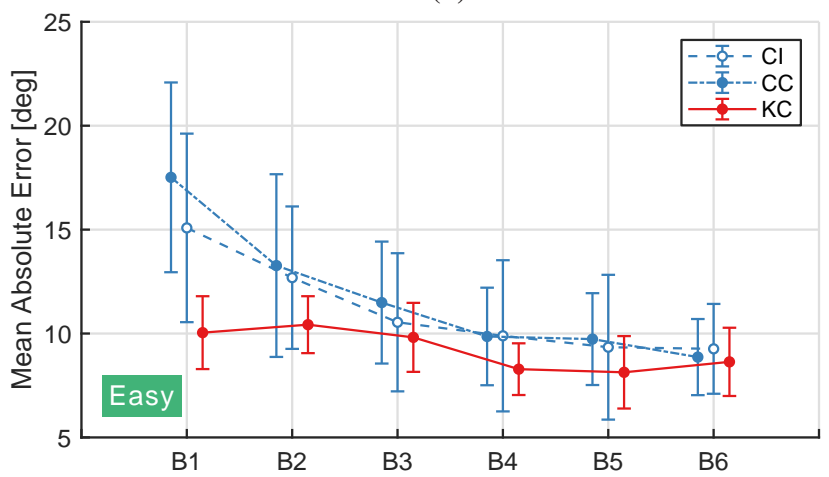

(b)

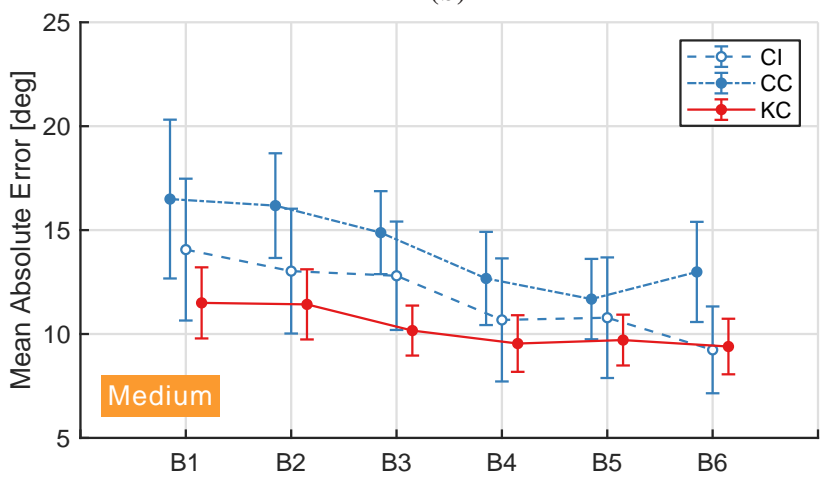

(c)

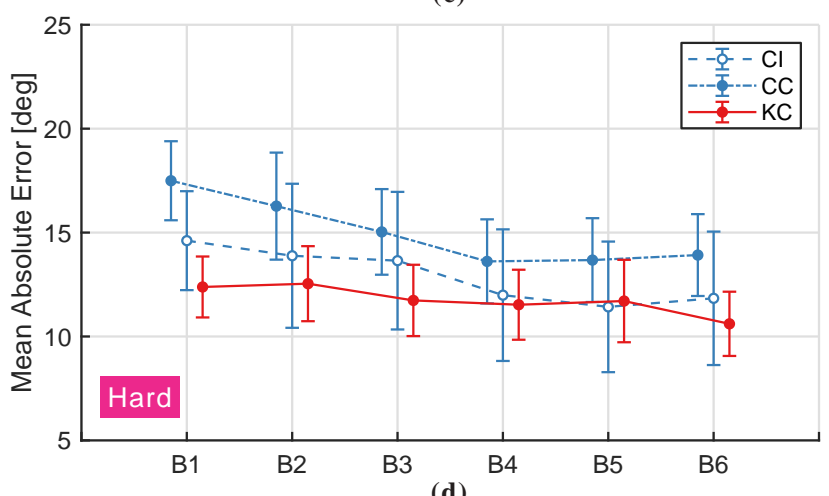

Figure 13. Results - Mean absolute error of subjects' trajectories (with error bars denoting the 95\% confidence interval) is depicted. (a) Averaging across block and difficulty, error plateaus at approximately 12 degrees. There is a statistical difference between conditions in B1, however by $\mathrm{B} 6$ no statistical difference is observable. We can see that cutaneous guidance required more learning since error under these conditions was initially higher and drops with a steeper slope than the kinesthetic guidance condition. (b) The plateau for error is lowest for Easy trajectories. (c) The learning curves for the Medium trajectory plateau higher than Easy but lower than (d) Hard which plateaus quickly.
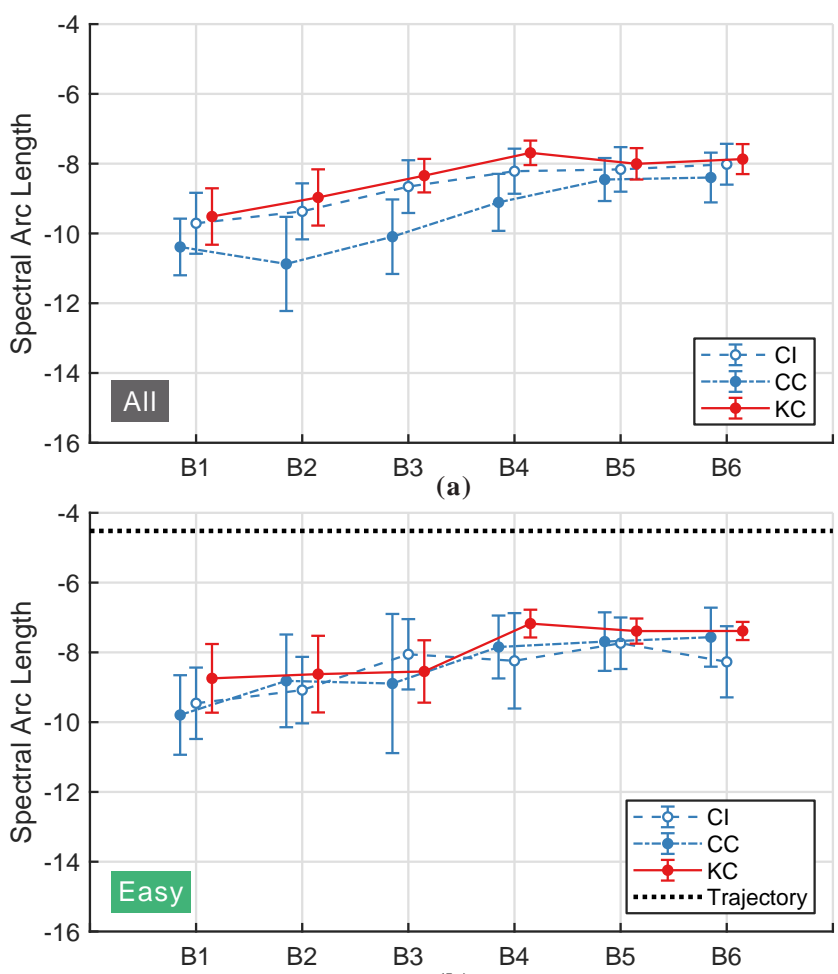

(b)

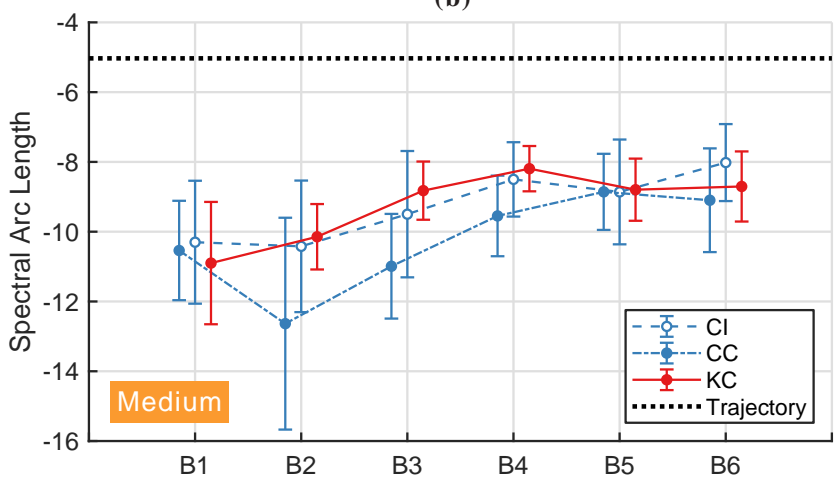

(c)

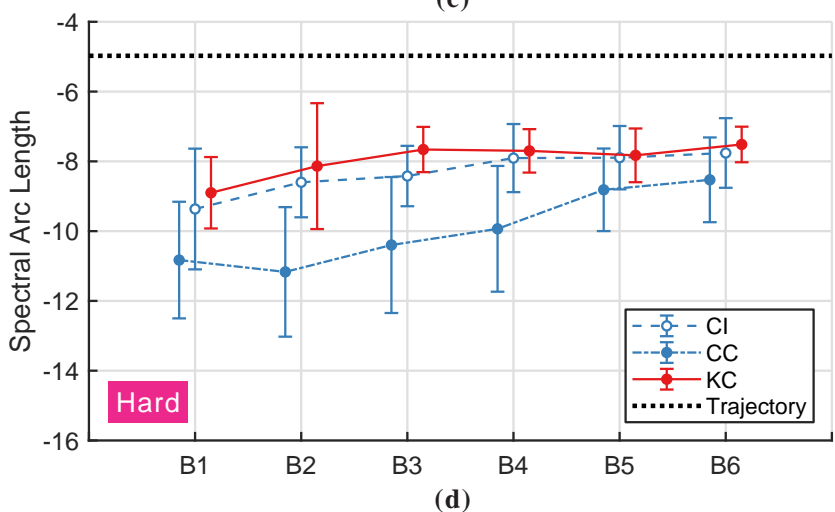

Figure 14. Spectral Arc Length (SPARC) of subjects' trajectories gives an indication of their movement smoothness. Error bars denote the $95 \%$ confidence interval. Smoother movements are represented by less negative values. The dotted black line represents the SPARC value of the actual trajectory (i.e. the best attainable smoothness value). (a) Observing all difficulties combined shows that conditions $\mathrm{Cl}$ and $\mathrm{KC}$ generally resulted in the same rate of smoothness improvement, while $\mathrm{CC}$ is slightly less smooth overall and shows higher variance, especially on (c) Medium and (d) Hard difficulties. Smoothness was similar for all conditions in the (b) Easy difficulty. 
and B6 to assess improvement over the course of the experiment. A one-way ANOVA was used for B1 and B6 individually, with additional contrasts to compare $\mathrm{CC}$ and $\mathrm{CI}$, as well as comparing $\mathrm{KC}$ against both $\mathrm{CC}$ and $\mathrm{CI}$.

\subsection{Results}

Fig. 12 shows the average trajectories for all subjects at the beginning (B1) and end (B6) of the experiment for each condition and difficulty. Of particular interest here are the considerably higher starting error and standard deviation of the cutaneous conditions ( $\mathrm{CI}$ and $\mathrm{CC}$ ) compared to the kinesthetic condition (KC). However, by B6, all conditions appear similar, especially for Easy trials. Also of note is that the contralateral cutaneous condition shows higher error than its ipsilateral counterpart.

The error over each Block is shown in Fig. 13 comparing each feedback condition as a whole and within each task difficulty. There was an effect across Block $(F(3.02,81.6)=39.88, \mathrm{p}<$ $.001)$ and Difficulty $(F(1.36,36.6)=12.3, \mathrm{p}<.001)$, though, interestingly, not across Condition as a main effect. Condition did have a significant interaction with Block $(F(6.04,81.6)=3.09$, $\mathrm{p}=.009)$, and is broken down further to assess learning and differences in the feedback conditions toward the beginning and end of the experiment. The overall error in the final (B6) and initial (B1) blocks were significantly different $(t(29)=6.8, \mathrm{p}<$ $.001)$, showing an overall improvement. At the beginning of the experiment, in $\mathrm{B} 1$, there was an effect of Condition $(F(1,27)=$ $342.0, \mathrm{p}<.001)$, and $\mathrm{KC}$ did have lower errors compared to cutaneous feedback, $\mathrm{CC}$ and $\mathrm{CI},(F(1,27)=7.70, \mathrm{p}=.01)$, though no significant difference between the contralateral and ipsilateral locations of the cutaneous feedback, CC and CI was found. These differences in feedback condition were not present by the end of the experiment, however, where $\mathrm{B} 6$ has no significant effect of Block, or distinction between $\mathrm{KC}$ and $\mathrm{CI}$ or $\mathrm{CC}$.

The results for smoothness are similar to those for mean absolute error with respect to learning, see Fig. 14, although it does not seem to be impacted by the feedback condition. The main effects show the Block $(F(2.89,78.16)=16.13, \mathrm{p}<.001)$ and Difficulty $(F(2,54)=18.0, \mathrm{p}<.001)$ have a significant effect, though no effect of Condition or interactions with Block or Difficulty. Indeed, no significant difference in smoothness was found between the feedback types within B6 or B1 individually. The smoothness of subject movements did significantly improve from B1 to B6, $(t(29)=6.24, \mathrm{p}<.001)$.

\section{Discussion}

\subsection{Feedback vs. Feedforward Guidance}

There are several interesting takeaways from the results of the first experiment (Section 4). Despite our initial assumption that feedback guidance would enable subjects to follow the trajectory more closely, it was actually the feedforward mode that proved most beneficial. This assumption was based on the fact that we, the authors, were actually quite good at interpreting feedback guidance in our initial pilot testing. While this may be generally true for experienced CUFF users, it is simply not the case for first time novices. We believe the reason for this is that in order for subjects to receive substantial cutaneous guidance cues in the feedback condition, they necessarily must make large errors. Once the trajectory is lost, subjects make frantic movements to regain it, further amplifying cutaneous feedback cues and thus confusion. It's possible that this effect could be mitigated with non-linear mappings so small errors result in higher levels of skin stretch than large errors. Even more telling is the fact that feedback guidance provided no benefit over using visuals alone, as evident by similar subject error and smoothness in Fig. 9 and 10, respectively. In fact, Fig. 15 reveals that the quartile distribution of subjects in the $\mathrm{V}$ and FB conditions are nearly identical for all difficulties.

It seems likely that feedforward guidance proved successful because subjects were more easily able to integrate the directional stretch cues with proximity feedback from the visuals (i.e. the pendulum light). However, it is also evident that feedforward guidance was mutually beneficial to visual feedback since subject performance was significantly better with than without guidance.

Ultimately, the best guidance mechanisms may combine aspects of both feedback and feedforward. For example, training may benefit from a feedforward only guidance scheme during early stages to familiarize inexperienced users, but implement feedback mechanisms progressively or in later stages to attain higher accuracy results. A hybrid system may use feedback guidance when a subject is far away from a desired trajectory, and switch to feedforward mechanisms when they become close. It may also be possible to combine both feedback and feedforward mechanisms simultaneously in multi-sensory or multi-DOF devices. As an example, feedforward guidance could be mapped to skin stretch, while proximity feedback could be mapped to squeeze or another cutaneous sensory channel.

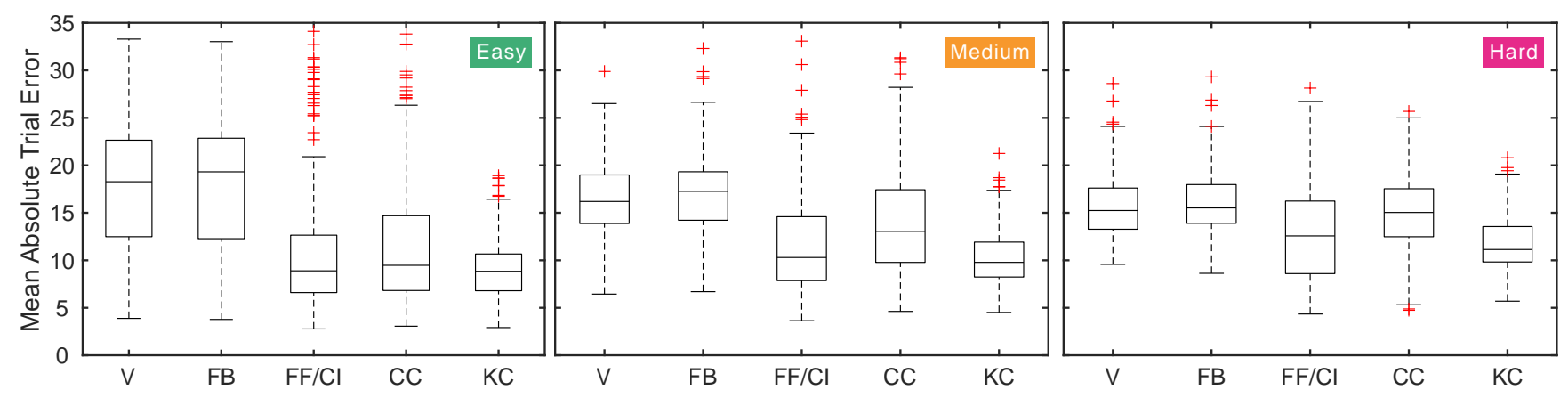

Figure 15. Boxplots of subjects' mean absolute trial error for all experimental conditions presented in Sections 4 and 5 Whiskers extend to the 1.5 inter-quartile range (IRQ) so that extreme values can be clearly seen. For all difficulties, feedback based cutaneous guidance (FB) shows a very similar distribution of performance when compared with the visuals only condition (V). Feedforward based cutaneous guidance (FF/Cl) shows an improvement in performance compared to both (FB) and (V). While the kinesthetic condition (KC) has more consistent subject performance compared to ipsilateral $(\mathbf{F F} / \mathbf{C l})$ and contralateral cutaneous guidance $(\mathbf{C C})$, no notable difference exists between $(\mathbf{F F} / \mathbf{C l})$ and $(\mathbf{C C})$ themselves. 


\subsection{Cutaneous vs. Kinesthetic Guidance}

Performance under kinesthetic guidance improved little over the course of the second experiment. This was expected, as people are more accustomed to interpreting proprioceptive information than cutaneous information, hlespecially in the context of guidance. The most interesting result is that performance under cutaneous guidance appears to converge towards that of kinesthetic guidance.

Error bars for cutaneous guidance conditions in Fig. 13 show a much higher degree of variance than the kinesthetic condition. Further investigation into the distribution of subject error is shown with the boxplots in Fig. 15 Here we can see that conditions CI and $\mathrm{CC}$ contain a larger number of extreme values outside their 1.5 IQR, and exhibit a much less uniform distribution than the $\mathrm{KC}$ condition. While many subjects were successful in interpreting cutaneous guidance, a handful were unable to. This suggests that either cutaneous guidance generally requires more practice, or that it is not a perfect guidance mechanism for all individuals.

Note that the $\mathrm{CI}$ and $\mathrm{KC}$ conditions generally share the same median value across all difficulties, shown by the histogram in Fig. 16 Again, we can see while the kinesthetic condition displays a mostly uniform distribution, the cutaneous condition is skewed toward the right-hand side by subjects with poor performance. However, the most interesting observation is that cutaneous guidance is more represented in the first four to five bins of trial error. This suggests that perhaps cutaneous guidance has a higher ceiling for guidance efficacy, and that experts receiving cutaneous guidance may outperform experts receiving kinesthetic guidance.

It is worth noting that there was no general consensus among subjects as to whether the mapping direction was "right." While some subjects felt that rotating their wrist in the same direction as skin stretch was intuitive, others felt that the mapping should have been inverted. It is unlikely that inverting cutaneous guidance would have changed overall mean performance across subjects, but there may be some benefit to allowing trainees to choose the directional mapping that is most intuitive to them.

Another common phenomenon found in cutaneous guidance is that some subjects easily "forgot" the directional mapping between skin stretch and wrist rotation, and often moved in the direction opposite of the guidance at the beginning of trials. This is most evident from the large standard deviation in the first period of Easy CI and CC trials for Block 6 (Fig 12). However, upon realizing that their interpretation was reversed, subjects were able to quickly correct themselves. Some subjects attributed this phenomenon to a lapse in focus, while others could not offer an explanation. While inconclusive, it may be that these subjects were also those who suggested the directional mapping should have been inverted. Common amongst most subjects was the opinion that perceiving stretch motion was relatively easy, but detecting directionality required more attentiveness.

It reasonable to conclude that, given enough time and training, individuals may be able to use cutaneous guidance nearly as effectively as kinesthetic guidance. This is an exciting result for situations in which separated guidance is required, but the costs or ease of providing kinesthetic guidance with a kinematically similar device is prohibitive. Further, we believe that cutaneous skin stretch guidance can be generalized to other tasks which require navigation, steering, or directional force application as long as the mapping between guidance and action remains intuitive. The CUFF and similar cutaneous devices can provide inexpensive and practical solutions to separating guidance that is easily integrated with the training protocol and adaptable to real world applications.

\subsection{Ipsilateral vs. Contralateral Cutaneous Guidance}

Separating cutaneous guidance into its ipsilateral and contralateral conditions seems to reveal that subjects performed better with ipsilateral guidance. Herein lies one of the major advantages of cutaneous guidance: unlike kinesthetic guidance, cutaneous guidance may be located anywhere, and as shown, placing it more proximally to the point of interaction may have benefits. This general observation compliments the work of Brown et. al [48], which concluded that co-locating feedback and action was more effective than non co-locating them. On the other hand, in training situations like robotic rehabilitation where ipsilateral guidance may be impossible due to sensory loss, it is reassuring to observe that contralateral guidance can also be effective.

\section{Conclusion ANd Future Work}

We have presented a novel approach to SSA through wearable cutaneous devices, and have directly compared this approach with a traditional kinesthetic approach. Task forces and guidance forces were separated, with task forces provided through an exoskeleton, and guidance forces provided either through a kinematically similar exoskeleton on the contralateral arm or a wearable skin stretch device on the ipsilateral or contralateral arm. While each condition promoted decreased error rates and improved movement smoothness as time progressed, comparisons of subject performance measures revealed a significant effect of guidance condition. Given enough time and training, individuals may be able to learn and use cutaneous guidance as effectively as kinesthetic guidance, offering a more harmonious integration
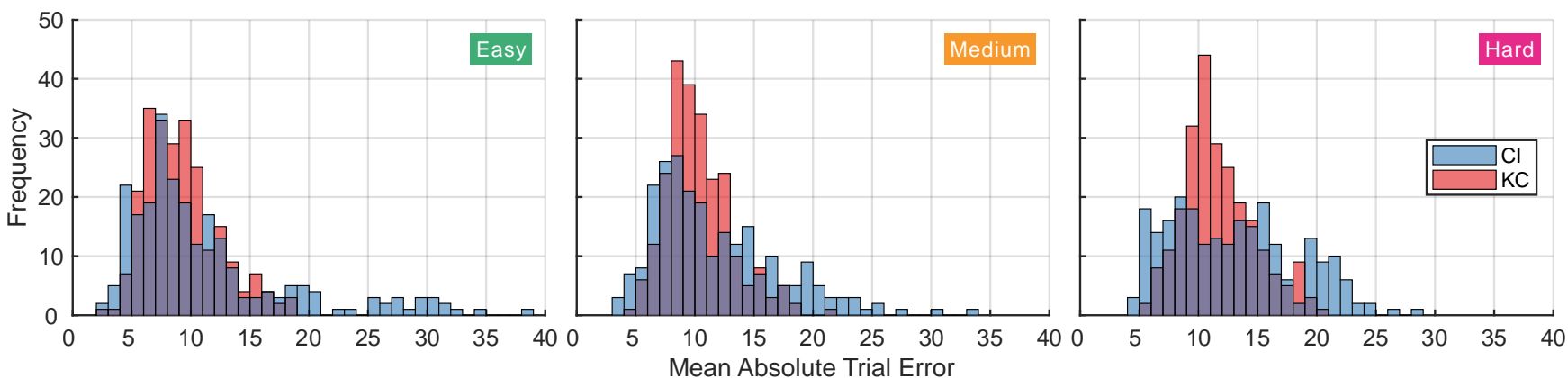

Figure 16. Histogram comparing the distributions of subject performance under the cutaneous ipsilateral condition $(\mathbf{C l})$ and the kinesthetic condition (KC). While the kinesthetic condition is more normally distributed at high levels of performance, the best subject performance occurred under the cutaneous ipsilateral condition, as seen in the first four to five bins on the left hand side. This may suggest that cutaneous guidance has a higher potential for improving trajectory accuracy than kinesthetic guidance. 
of haptic guidance with the user's body and a more economic, safer, and generalizable approach than those offered by prohibitive kinesthetic devices. Overall, our results are very promising since they suggest a novel way of providing haptic feedback in SSA through wearable cutaneous devices.

The results presented here suggest a few paths for further investigation. First and of highest importance is exploring the applicability of spatially separated cutaneous guidance to learning new tasks, and whether or not learned skills will be retained and/or transfer to similar tasks. While our task design showed that cutaneous guidance is effective in providing guidance and reducing error, it is not necessarily conclusive that it accelerated learning rates (as suggested by the similarity in slopes of the visual and cutaneous conditions in Fig. 97. We believe that part of the reason for this is that our task did not place enough importance on subjects understanding task forces. While the forces arising from the pendulum were certainly felt and required stabilization, subjects didn't necessarily have to develop an internal model of their dynamics to complete the task. In other words, our task dynamics were little more than random disturbance forces. One possibly interesting take on the current task is to require subjects to invert and balance the pendulum, as originally studied by Gillespie [22]. Relatedly, stick balancing has been extensively studied in humans, and is known to be a task that can be learned and trained [49]. Pendulum inversion and balancing also offers the ability to test for learning that occurs through the use of both forms of motor control - feedforward control, as is necessary for inversion, and feedback control, necessary for balancing [50].

Secondly, more investigation should be made into the differences between novice and experts users of cutaneous guidance. Specifically, why do expert users seem to interpret feedback based guidance with ease while novices struggle, and how long does it take for novices to become experts? The final area of future research is in application of cutaneous SSA to real-world haptic training. We believe the most immediate path forward will be in integrating cutaneous guidance with already existing haptic simulators for surgical training, but we are also excited for its potential use in robotic rehabilitation.

\section{ACKNOWLEDGMENTS}

This research has received funding from the National Science Foundation IGERT fellowship (NSF DGE-1250104), and from the European Union's Horizon 2020 Research and Innovation Programme under Grant Agreement No.688857 (SoftPro). The authors thank Jennifer Sullivan for her guidance on the analysis.

\section{REFERENCES}

[1] R. Sigrist, G. Rauter, R. Riener, and P. Wolf, "Augmented visual, auditory, haptic, and multimodal feedback in motor learning: A review," Psychonomic Bulletin \& Review, vol. 20, no. 1, pp. 21-53, 2013.

[2] L. Marchal-Crespo and D. J. Reinkensmeyer, "Review of control strategies for robotic movement training after neurologic injury," Journal of Neuroengineering and Rehabilitation, vol. 6, no. 1, p. 20, 2009.

[3] K. S. Hale and K. M. Stanney, "Deriving haptic design guidelines from human physiological, psychophysical, and neurological foundations," IEEE Comput. Graphics and Appl., vol. 24, no. 2, pp. 33-39, 2004.

[4] W. H. Jantscher, S. Pandey, P. Agarwal, S. H. Richardson, B. R. Lin, M. D. Byrne, and M. K. O'Malley, "Toward improved surgical training: Delivering smoothness feedback using haptic cues." San Francisco, CA: IEEE, 03/2018 2018, pp. 241-246.

[5] D. Escobar-Castillejos, J. Noguez, L. Neri, A. Magana, and B. Benes, "A review of simulators with haptic devices for medical training," Journal of Medical Systems, vol. 40, no. 4, pp. 1-22, 2016.
[6] E. M. Overtoom, T. Horeman, F.-W. Jansen, J. Dankelman, and H. W. R. Schreuder, "Haptic feedback, force feedback, and force-sensing in simulation training for laparoscopy: A systematic overview," Journal of Surgical Education, 2018.

[7] P. Lum, S. Godfrey, E. Brokaw, R. Holley, and D. Nichols, "Robotic approaches for rehabilitation of hand function after stroke," American Journal of Physical Medicine \& Rehab., vol. 91, no. 11, pp. S242-S254, 2012.

[8] A. D Steinberg, P. G Bashook, J. Drummond, S. Ashrafi, and M. Zefran, "Assessment of faculty perception of content validity of periosim, a haptic-3d virtual reality dental training simulator," Journal of Dental Education, vol. 71, pp. 1574-82, 2008.

[9] N. Gavish, T. Gutiérrez, S. Webel, J. Rodríguez, M. Peveri, U. Bockholt, and F. Tecchia, "Evaluating virtual reality and augmented reality training for industrial maintenance and assembly tasks," Interactive Learning Environments, vol. 23, no. 6, pp. 778-798, 2015.

[10] S. Payandeh and Z. Stanisic, "On application of virtual fixtures as an aid for telemanipulation and training," in Proceedings 10th Symposium on Haptic Interfaces for Virtual Environment and Teleoperator Systems, 2002, pp. 18-23.

[11] C. Bermejo and P. Hui, "A survey on haptic technologies for mobile augmented reality," CoRR, 2017.

[12] L. M. Crespo and D. J. Reinkensmeyer, "Effect of robotic guidance on motor learning of a timing task," in Intl. Conf. on Biomedical Robotics and Biomechatronics, 2008, pp. 199-204.

[13] L. Crespo and D. Reinkensmeyer, "Haptic guidance can enhance motor learning of a steering task." Journal of Motor Behavior, vol. 40 6, pp. 545-56, 2008.

[14] D. Powell and M. K. O'Malley, "The task-dependent efficacy of sharedcontrol haptic guidance paradigms," IEEE Transactions Haptics, vol. 5, pp. 208-219, 2012.

[15] R. A. Scheidt, D. J. Reinkensmeyer, M. A. Conditt, W. Z. Rymer, and F. A. Mussa-Ivaldi, "Persistence of motor adaptation during constrained, multi-joint, arm movements." Journal of Neurophysiology, vol. 84 2, pp. $853-62,2000$.

[16] K. A. Thoroughman and R. Shadmehr, "Learning of action through adaptive combination of motor primitives," Nature, vol. 407, pp. 742$747,2000$.

[17] S. A. Bowyer, B. L. Davies, and F. Rodriguez y Baena, "Active constraints/virtual fixtures: A survey," IEEE Transactions on Robotics, vol. 30, no. 1, pp. 138-157, 2014.

[18] J. L. Patton et al., "Evaluation of robotic training forces that either enhance or reduce error in chronic hemiparetic stroke survivors," $E x$ perimental Brain Research, vol. 168, pp. 368-383, 2005.

[19] M. K. O'Malley, A. Gupta, M. Gen, and Y. Li, "Shared control in haptic systems for performance enhancement and training," J. Dynamic Systems, Measurement, and Control, vol. 128, no. 1, pp. 75-85, 2006.

[20] T. Endo, H. Kawasaki, K. Kigaku, and T. Mouri, "Transfer method of force information using five-fingered haptic interface robot," in Second Joint EuroHaptics Conference and Symposium on Haptic Interfaces for Virtual Environment and Teleoperator Systems (WHC'07), 2007, pp. 599-600.

[21] J. Ahn and N. Hogan, "Feasibility of dynamic entrainment with ankle mechanical perturbation to treat locomotor deficit," in 2010 Annual International Conference of the IEEE Engineering in Medicine and Biology, 2010, pp. 3422-3425.

[22] R. B. Gillespie, M. O’Modhrain, P. Tang, D. Zaretzky, and C. Pham, "The virtual teacher," in Proceedings of the ASME Dynamic Systems and Control Division, vol. 64, 1998, pp. 171-178.

[23] G. Wulf, C. Shea, and C. A. Whitacre, "Physical-guidance benefits in learning a complex motor skill," Journal of Motor Behavior, vol. 30, pp. 367-80, 1998

[24] D. Nozaki, I. Kurtzer, and S. H. Scott, "Limited transfer of learning between unimanual and bimanual skills within the same limb," Nature Neuroscience, vol. 9, no. 11, pp. 1364-1366, 2006.

[25] L. Tcheang, P. M. Bays, J. N. Ingram, and D. M. Wolpert, "Simultaneous bimanual dynamics are learned without interference," Experimental Brain Research, vol. 183, no. 1, pp. 17-25, 2007.

[26] G. Rauter, J. von Zitzewitz, A. Duschau-Wicke, H. Vallery, and R. Riener, "A tendon-based parallel robot applied to motor learning in sports," in Intl. Conf. on Biomedical Robotics and Biomechatronics, 2010, pp. 82 87.

[27] J. von Zitzewitz, P. Wolf, V. Novaković, M. Wellner, G. Rauter, A. Brunschweiler, and R. Riener, "Real-time rowing simulator with multimodal feedback," Sports Technology, vol. 1, no. 6, pp. 257-266, 2008.

[28] L. Marchal-Crespo, M. van Raai, G. Rauter, P. Wolf, and R. Riener, "The effect of haptic guidance and visual feedback on learning a complex 
tennis task," Experimental Brain Research, vol. 231, no. 3, pp. 277-291, 2013.

[29] Y. Li, V. Patoglu, and M. K. O’Malley, "Negative efficacy of fixed gain error reducing shared control for training in virtual environments," $A C M$ Transactions on Applied Perception (TAP), vol. 6, no. 1, p. 3, 2009.

[30] L. M. Crespo and D. J. Reinkensmeyer, "Haptic guidance can enhance motor learning of a steering task," Journal of Motor Behavior, vol. 40, no. 6, pp. 545-557, 2008.

[31] C. Pacchierotti, S. Sinclair, M. Solazzi, A. Frisoli, V. Hayward, and D. Prattichizzo, "Wearable haptic systems for the fingertip and the hand: Taxonomy, review, and perspectives," IEEE Transactions Haptics, 2017.

[32] M. Bianchi, "A fabric-based approach for wearable haptics," Electronics, vol. 5 , no. 3, p. 44, 2016.

[33] A. A. Stanley and K. J. Kuchenbecker, "Evaluation of tactile feedback methods for wrist rotation guidance," IEEE Transactions Haptics, vol. 5, no. 3, pp. 240-251, 2012.

[34] K. Bark, E. Hyman, F. Tan, E. Cha, S. A. Jax, L. J. Buxbaum, and K. J. Kuchenbecker, "Effects of vibrotactile feedback on human learning of arm motions," IEEE Transactions Neural Syst. and Rehabil. Eng., vol. 23, no. 1, pp. 51-63, 2015.

[35] S. L. Norman, A. J. Doxon, B. T. Gleeson, and W. R. Provancher, "Planar hand motion guidance using fingertip skin-stretch feedback," IEEE Transactions Haptics, vol. 7, no. 2, pp. 121-130, 2014.

[36] E. Ruffaldi, A. Filippeschi, A. Frisoli, O. Sandoval, C. A. Avizzano, and M. Bergamasco, "Vibrotactile perception assessment for a rowing training system," in EuroHaptics Conf. and Symposium on Haptic Interfaces for Virtual Environment and Teleoperator Systems., 2009, pp. 350-355.

[37] C. Pacchierotti, M. Abayazid, S. Misra, and D. Prattichizzo, "Teleoperation of steerable flexible needles by combining kinesthetic and vibratory feedback," IEEE Transactions Haptics, vol. 7, no. 4, pp. 551-556, Oct 2014.

[38] S.-C. Lim, H.-K. Lee, and J. Park, "Role of combined tactile and kinesthetic feedback in minimally invasive surgery," The International Journal of Medical Robotics and Computer Assisted Surgery, vol. 11, no. 3, pp. 360-374, 2015.

[39] A. R. Peon, C. Pacchierotti, and D. Prattichizzo, "Vibrotactile stimuli for augmented haptic feedback in robot-assisted surgery," in 2013 World Haptics Conference (WHC), April 2013, pp. 473-478.

[40] K. Kim and J. E. Colgate, "Haptic feedback enhances grip force control of semg-controlled prosthetic hands in targeted reinnervation amputees," IEEE Transactions Neural Syst. and Rehabil. Eng., vol. 20, no. 6, pp. 798-805, 2012.

[41] E. Pezent, S. Fani, J. Bradley, M. Bianchi, and M. K. O’Malley, "Separating haptic guidance from task dynamics: A practical solution via cutaneous devices," in IEEE Haptics Symposium, 2018, pp. 20-25.

[42] E. Pezent, C. G. Rose, A. D. Deshpande, and M. K. O’Malley, "Design and characterization of the OpenWrist: A robotic wrist exoskeleton for coordinated hand-wrist rehabilitation," in IEEE Intl. Conf. Rehabil. Robot. (ICORR), 2017.

[43] S. Casini, M. Morvidoni, M. Bianchi, M. Catalano, G. Grioli, and A. Bicchi, "Design and realization of the cuff-clenching upper-limb force feedback wearable device for distributed mechano-tactile stimulation of normal and tangential skin forces," in IEEE Intl. Conf. Intell. Robots and Syst. (IROS), 2015, pp. 1186-1193.

[44] J. A. French, C. G. Rose, and M. K. O’Malley, "System characterization of MAHI Exo-II: A robotic exoskeleton for upper extremity rehabilitation," in ASME Dynamic Systems and Control Conf., 2014.

[45] S. B. Godfrey, M. Bianchi, A. Bicchi, and M. Santello, "Influence of force feedback on grasp force modulation in prosthetic applications: A preliminary study," in IEEE Intl. Conf. Eng. in Medicine and Biology Society $($ EMBC), 2016, pp. 5439-5442.

[46] S. Fani, S. Ciotti, M. Catalano, G. Grioli, A. Tognetti, G. Valenza, A. Ajoudani, and M. Bianchi, "Simplifying telerobotics: Wearability and teleimpedance improves human-robot interactions in teleoperation," IEEE Robotics Automation Magazine, vol. 25, no. 1, pp. 77-88, 2018.

[47] S. Balasubramanian, A. Melendez-Calderon, A. Roby-Brami, and E. Burdet, "On the analysis of movement smoothness," Journal of NeuroEngineering and Rehabil., vol. 12, no. 1, p. 112, 2015.

[48] J. D. Brown, R. B. Gillespie, D. Gardner, and E. A. Gansallo, "Colocation of force and action improves identification of force-displacement features," in IEEE Haptics Symposium, 2012, pp. 187-193.

[49] R. Balasubramaniam, "On the control of unstable objects: The dynamics of human stick balancing," in Progress in Motor Control, M. J. Richardson, M. A. Riley, and K. Shockley, Eds. New York, NY: Springer New York, 2013, pp. 149-168.

[50] E. Burdet, D. Franklin, and T. Milner, Human Robotics: Neuromechanics and Motor Control. MIT Press, 2013.

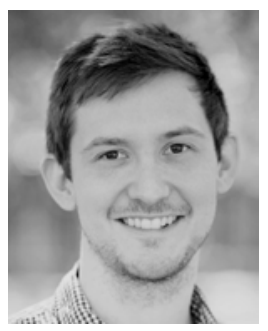

Evan Pezent (S'16) received the B.S. degree from The University of Alabama in 2014 and the M.S. degree from Rice University in 2017. He is currently a Ph.D. student and NSF IGERT fellow in the Mechatronics and Haptic Interfaces Lab at Rice University. His research investigates how haptic devices can accelerate training outcomes and enhance believability and immersion in augmented and virtual reality (AR/VR). In 2018, he spent six months at Facebook Reality Labs developing wearable haptic devices for AR/VR.

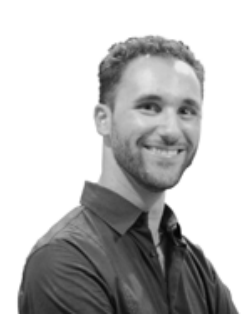

Simone Fani (S'17) received the B.S. degree in information engineering from the University of Siena in 2011, and the M.S. degree in robotics and automation engineering from the University of Pisa in 2015. He is currently working toward the Ph.D. degree at the Research Center "E. Piaggio", University of Pisa. His research interests include design and validation of haptic interfaces for prosthetics and teleoperation systems, medical and rehabilitation robotics, and mathematical modeling of the sense of touch.

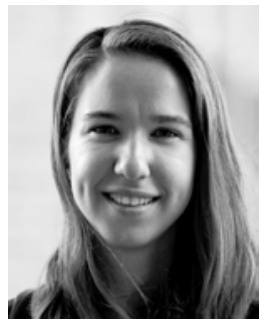

Janelle Clark (S'16) received her B.S. degree in Mechanical Engineering from Clarkson University in 2015 and her M.S. degree in Mechanical Engineering from Rice University in 2017. She is currently a Ph.D. student in the Mechatronics and Haptics Interfaces Lab at Rice University. Her research interests include the assessment of passive haptic stimuli and coupled control of mechanical and electrical systems for haptic applications in teleoperation, proprioception for upper-limb prostheses.

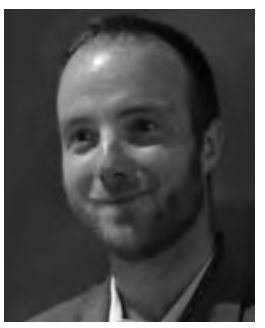

Matteo Bianchi (M'12) received from the Università di Pisa the B.S degree and the M.S degree in Biomedical Engineering (both cum laude), and the Ph.D. in Automatics, Robotics, Bioengineering, in 2004, 2007 and 2012, respectively. From January to June 2011, he was visiting student at the Johns Hopkins University. Currently, he is a tenure track Assistant Professor of Automatic Controls and Robotics at the Research Centre "E. Piaggio" and the Department of Information Engineering of the Università di Pisa, and clinical research affiliate at Mayo Clinic. His research interests include robotic/human hands and manipulation; haptic interfaces and tactile sensing for advanced Human-Machine Interaction. $\mathrm{He}$ is author of more than 100 peer-reviewed contributions and serves as member of the editorial/organizing board of international conferences and journals. He is the editor of the book Human and Robot Hands. $\mathrm{He}$ is recipient of several awards, including the JCTF novel technology paper award (IROS 2012) and the Best Paper Award (IEEE-RAS Haptics Symposium 2016).

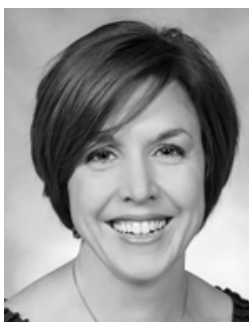

Marcia K O'Malley (SM'13) received the B.S. degree from Purdue University, West Lafayette, IN, USA, in 1996, and the M.S. and Ph.D. degrees from Vanderbilt University, Nashville, TN, USA, in 1999 and 2001, respectively, all in mechanical engineering. She is currently the Stanley C. Moore Professor of mechanical engineering, computer science, and electrical and computer engineering at Rice University, Houston, TX, USA, and directs the Mechatronics and Haptic Interfaces Laboratory. She is currently an Adjunct Faculty in the Departments of Physical Medicine and Rehabilitation at the Baylor College of Medicine, Houston, TX, USA, and the University of Texas Medical School at Houston, Houston, TX, USA, and the Director of Rehabilitation Engineering at TIRR-Memorial Hermann Hospital, Houston, TX, USA. Her research addresses issues that arise when humans physically interact with robotic systems, with a focus on training and rehabilitation in virtual environments. 